\title{
A volume-fraction based algorithm for hybrid barotropic and non-barotropic two-fluid flow problems
}

Keh-Ming Shyue

Department of Mathematics, National Taiwan University, Taipei 106, Taiwan

Received

\begin{abstract}
The aim of this paper is to describe a simple Eulerian interface-capturing approach for the efficient numerical resolution of a hybrid barotropic and non-barotropic two-fluid flow problem in more than one space dimension. We use the compressible Euler equations as a model system with the thermodynamic property of each of the barotropic and non-barotropic fluid components characterized by the Tait and Noble-Abel equations of state, respectively. The algorithm is based on a volume fraction formulation of the equations together with an extended equation of state that is devised to give an approximate treatment for the mixture of more than one fluid component within a grid cell. A standard high-resolution wave propagation method is employed to solve the proposed two-fluid model with the dimensional-splitting technique incorporated in the method for multidimensional problems. Several numerical results are presented in one and two space dimensions that show the feasibility of the algorithm as applied to a reasonable class of practical problems without the occurrence of any spurious oscillation in the pressure near the smeared material interfaces. This includes, in particular, solutions for a study on the variation of the jet velocity with the incident shock pressure arising from the collapse of an air cavity in water under a shock wave.
\end{abstract}

Key words: Volume-fraction based algorithm, Tait equation of state, Noble-Abel equation of state, Gascavity collapse, Jetting

PACS: $02.70 . \mathrm{Bf}, 46.15 .-\mathrm{x}, 47.11 .+\mathrm{j}, 47.55 . \mathrm{Kf}$

\section{Introduction}

This paper is concerned with a simplified compressible two-phase flow problem where there is a material interface separating regions of two different barotropic and non-barotropic fluid components within an $N_{d} \geq 1$ spatial domain. In this problem, the flow regime of interest is assumed to be homogeneous with no jumps in the pressure and velocity (the normal component of it) across the interface, and the physical effects such as the viscosity, surface tension, and heat conduction are assumed to be small, and hence can be ignored in the problem formulation. With that, we use an Eulerian viewpoint of the model system, that on the barotropic part of the domain, the fluid is governed by the isentropic version of the compressible Euler equations as

$$
\frac{\partial}{\partial t}\left[\begin{array}{c}
\rho \\
\rho u_{i}
\end{array}\right]+\sum_{j=1}^{N_{d}} \frac{\partial}{\partial x_{j}}\left[\begin{array}{c}
\rho u_{j} \\
\rho u_{i} u_{j}+p(\rho) \delta_{i j}
\end{array}\right]=0
$$

for $i=1,2, \cdots, N_{d}$, while on the non-barotropic part of the domain, the fluid is governed by the full set of the

Correspondence to: K.-M. Shyue

(e-mail: shyue@math.ntu.edu.tw) compressible Euler equations as

$$
\frac{\partial}{\partial t}\left[\begin{array}{c}
\rho \\
\rho u_{i} \\
\rho E
\end{array}\right]+\sum_{j=1}^{N_{d}} \frac{\partial}{\partial x_{j}}\left[\begin{array}{c}
\rho u_{j} \\
\rho u_{i} u_{j}+p \delta_{i j} \\
\rho E u_{j}+p u_{j}
\end{array}\right]=0 .
$$

Here $\rho, u_{j}, p, E$, and $\delta_{i j}$ denote the density, the particle velocity in the $x_{j}$-direction, the pressure, the specific total energy, and the Kronecker delta, respectively.

To complete the model, in this work, the constitutive law for each of the barotropic and non-barotropic components is taken to satisfy the Tait equation of state for compressible liquids (or called the Murnaghan equation of state in the context of an elastic solid [25]),

$$
p(\rho)=\left(p_{0}+\mathcal{B}\right)\left(\frac{\rho}{\rho_{0}}\right)^{\gamma}-\mathcal{B},
$$

and the Noble-Abel equation of state for real gases (or called the constant covolume equation of state [40]),

$$
p(\rho, e)=\left(\frac{\gamma-1}{1-b \rho}\right) \rho e
$$

in a respective manner. Note that in the above expressions $e$ represents the internal energy, $\rho_{0}$ and $p_{0}$ represent the density and pressure at a reference state, $\mathcal{B}$ is a 
pressure-like constant, $b$ represents a finite size of volume occupied by molecules $(0 \leq b<1 / \rho)$, and $\gamma$ is the adiabatic constant $(\gamma>1)$, see $[20,23,27,45]$ for a typical set of data of practical importance. As usual, we have $E=e+\sum_{j=1}^{N_{d}} u_{j}^{2} / 2$. Sample applications of this twofluid model are, for instance, to the simulation of a shockinduced collapse of gas cavities in liquid $[7,8]$, or to the simulation of an underwater explosion bubble $[21,44]$.

For an efficient numerical resolution of this hybrid barotropic and non-barotropic two-fluid flow problem, we want to use a generalization of the classical shockcapturing method designed originally for single component flows in an Eulerian framework. It is known in the literature that the principal difficulty in the usual modification is the occurrence of spurious pressure oscillations when two or more fluid components are present in a grid cell. In the case of a barotropic or non-barotropic multicomponent problem, there has been quite a few numerical methods developed for that matter, see [1-3, $9,14,15,31,43]$ and the references cited therein for more exposition. In the current interest of the problems consisting of separate barotropic and non-barotropic regions, there is, however, a relatively few attempts devoted to the subject, see [5,21,37] for an example using a somewhat complicated Lagrangian-type approach as compared to the current Eulerian one.

The basic approach we take here is an extension of the previous work advocated by the author $[33,34,36]$ in that a mixture equation of state is introduced first as a basis to the modeling of the mixing between two different barotropic and non-barotropic fluid components. Then with the help of a volume-fraction function, we define an extended equation of state so that the pressure of the fluid can be determined explicitly no matter what fluid component (pure or not) is within a grid cell (see Section 2.1). Having that, as before, we are able to derive a volumefraction based of the model system that consists of the full Euler equations for the basic conserved variables and an additional set of equations for the volume fraction as well as the partial density of any one of the two fluid components in the problem. In our method, the latter equations are included in the algorithm primarily for an easy determination of the approximate location of the interface and also the computation of the pressure from the equation of state. It is important to note that these equations are put in a form so as to ensure a consistent modeling of the energy equation near the smeared interfaces, and also the fulfillment of the mass equation in the other single component regions (see Section 2.2).

To find approximate solutions of our model system, we use a high-order Godunov method based on a wavepropagation formulation with the dimensional-splitting technique incorporated in the method for multidimensional problems. Numerical results to be presented in Section 4 show that this is a viable approach to a reasonable class of practical problems in one and two space dimensions, without producing any wrong oscillations in the pressure near the interfaces.
It should be emphasized that the methodology we have proposed here is by no means limited to the two-fluid flows with the above chosen equations of state. Extension of the algorithm to problems involving more than two fluid components and more complicated equations of state, can be made in a straightforward manner by following the idea described in this paper and the five equation model of $\mathrm{Al}-$ laire et al. [3] and Massoni et al. [24]. Without going into the details for that, our goal is to establish the basic solution strategy and validate its use via some sample numerical experimentations.

The format of this paper is as follows. In Section 2, we describe the basic mathematical equations in a fluidmixture form that will be used later in a numerical method for constructing approximate solutions of our model hybrid barotropic and non-barotropic two-fluid problems. In Section 3, we give a brief review of the HLLC approximate Riemann solver and the high-resolution wave-propagation methods for solving problems in one and more than one space dimensions. Numerical results of some sample examples are shown in Section 4.

\section{Mathematical formulation}

\subsection{Equations of state}

To start out, we want to build a mixture equation of state that is prerequisite in our approach for modeling the numerical mixing between the barotropic and non-barotropic fluid components within a grid cell. The first step, we take here, is to assume that thermodynamically the mixture of the fluids characterized by (3) and (4) behaves like a typical non-barotropic fluid. Then using the first and second laws of thermodynamics, we may rewrite (3) and (4) in terms of the specific entropy $S$ and the specific volume $V=1 / \rho$ as

$$
p(V, S)=\mathcal{A}(\mathcal{S})\left(p_{0}+\mathcal{B}\right)\left(\frac{V_{0}}{V}\right)^{\gamma}-\mathcal{B},
$$

and

$$
p(V, S)=\mathcal{A}(S) p_{0}\left(\frac{V_{0}-b}{V-b}\right)^{\gamma},
$$

for each in turn, where $\mathcal{A}(S)=\mathcal{R} \exp \left[\left(S-S_{0}\right) / C_{V}\right]$ (cf. [10]). Here $C_{V}$ represents the specific heat at constant volume, $\mathcal{R}$ is the universal gas constant, and $S_{0}$ is the specific entropy at a reference state. As in the previous work for multicomponent problems with a van der Waals equation of state [33], we assume further that all the fluid components under concerned is in an adiabatic equilibrium with the same entropy, and accordingly we propose to define an equation of state for the fluid mixture that combines (5) with (6) to be the form

$$
p(V, S)=\mathcal{A}(\mathcal{S})\left(p_{0}+\mathcal{B}\right)\left(\frac{V_{0}-b}{V-b}\right)^{\gamma}-\mathcal{B} .
$$

Notice that, with the use of this so to be called modified Noble-Abel equation of state (7), we recover not only 
the case (6) when $\mathcal{B} \rightarrow 0$, but also the case (5) and so (3) as we set $S=S_{0}$ in $(5)$, when $b \rightarrow 0$. In the non-limiting case, however, where none of the material-dependent quantities $\mathcal{B}$ and $b$ is close to zero, $(7)$ does give a way to the representation of the cases in between, that is to the mixing of the present barotropic and non-barotropic components.

It should be mentioned that, as a practical matter for many fluid flow problems away from cavitation (which is the situation studied here), rather than using (7) for the fundamental thermodynamic description of the fluid mixture, it is customary to formulate the equation in a way with the often-used variables in compressible hydrodynamics: $\rho$ and $e$ instead. Again, by employing the basic thermodynamic principles, this can be done quite easily, leading to

$$
p(\rho, e)=\left(\frac{\gamma-1}{1-b \rho}\right)(\rho e-\mathcal{B})-\mathcal{B} .
$$

As to the computation of the fluid temperature $T$ which is important in a wide variety of applications, we may use one of the formulas,

$$
\begin{aligned}
& p(\rho, T)=\frac{\rho \mathcal{R} T}{1-b \rho}-\mathcal{B}, \\
& e(\rho, T)=\frac{\mathcal{R} T}{\gamma-1}+\frac{\mathcal{B}}{\rho},
\end{aligned}
$$

for realization.

Now, for an easy identification of the type of fluid component within a grid cell, a volume-fraction function $\alpha$ is introduced here for that purpose. For example, when grid cells contain only the barotropic component we may set $\alpha=1$, and so when grid cells contain only the nonbarotropic component we set $\alpha=0$. In case there are some cells cut by the interfaces where $\alpha \in(0,1)$, we then have both of these components occupied by the volume fractions $\alpha$ and $1-\alpha$ for each separately. With this definition of $\alpha$, the pressure of our two-fluid flow problem in all the fluid-component scenarios within a grid cell can be determined straightforwardly by

$$
p=\left\{\begin{array}{rrr}
\left(p_{0}+\mathcal{B}\right)\left(\frac{\rho}{\rho_{0}}\right)^{\gamma}-\mathcal{B} \text { if } \quad \alpha=1 \\
\left(\frac{\gamma-1}{1-b \rho}\right)(\rho e-\mathcal{B})-\mathcal{B} \text { if } \quad \alpha \neq 1,
\end{array}\right.
$$

provided that all the variables appeared there are defined and known a priori. Finally, it should be remarked that, in this work, the thermodynamical description of the materials of interest is limited by the stability requirement that the speed of sound of the fluid belongs to a set of real numbers.

\subsection{Equations of motion}

With the proposed constitutive law (9), it is clear that in regions where $\alpha=0$ or $\alpha=1$, there is no problem in using (1) and (2), respectively, for the complete description of the behavior of the underlying single-component flow. In regions where $\alpha \in(0,1)$, since the fluid mixture is modeled in a non-barotropic manner, it is quite common to apply (2) as a model system that describes the motion of the mixtures of the basic conserved variables. Besides that, as in our previous work on numerical methods for compressible multicomponent problems (cf. [32,33,35, 36]), we also bring in a set of effective equations for the problem-dependent material quantities so that the pressure can be computed easily from the equation of state.

\subsection{1 $\gamma$-based effective equations}

To derive the aforementioned effective equations for the mixture of material quantities in the current hybrid barotropic and non-barotropic flow application, we start with an interface-only problem as usual where both the pressure and each component of the particle velocities are constants in the domain, while the other variables such as the density and the material quantities are having jumps across some interfaces. Then, from (2), it is easy to obtain equations for the time-dependent behavior of the density and total internal energy as

$$
\begin{array}{r}
\frac{\partial \rho}{\partial t}+\sum_{j=1}^{N_{d}} u_{j} \frac{\partial \rho}{\partial x_{j}}=0, \\
\frac{\partial}{\partial t}(\rho e)+\sum_{j=1}^{N_{d}} u_{j} \frac{\partial}{\partial x_{j}}(\rho e)=0,
\end{array}
$$

in a respective manner. Now, by inserting the modified Noble-Abel equation of state (8) into the latter one, we find an alternative description of the energy equation

$$
\begin{aligned}
& \frac{\partial}{\partial t}\left(\frac{1-b \rho}{\gamma-1} p+\frac{\gamma-b \rho}{\gamma-1} \mathcal{B}\right)+ \\
& \quad \sum_{j=1}^{N_{d}} u_{j} \frac{\partial}{\partial x_{j}}\left(\frac{1-b \rho}{\gamma-1} p+\frac{\gamma-b \rho}{\gamma-1} \mathcal{B}\right)=0
\end{aligned}
$$

that is in relation to not only the pressure, but also the density and the material quantities: $\gamma, b$, and $\mathcal{B}$.

In our algorithm, to maintain the pressure in equilibrium as it should be for this interface-only problem, we split (10) into the following two equations for the fluid mixtures $(1-b \rho) /(\gamma-1)$ and $(\gamma-b \rho) \mathcal{B} /(\gamma-1)$ as

$$
\begin{aligned}
\frac{\partial}{\partial t}\left(\frac{1-b \rho}{\gamma-1}\right)+\sum_{j=1}^{N_{d}} u_{j} \frac{\partial}{\partial x_{j}}\left(\frac{1-b \rho}{\gamma-1}\right) & =0, \\
\frac{\partial}{\partial t}\left(\frac{\gamma-b \rho}{\gamma-1} \mathcal{B}\right)+\sum_{j=1}^{N_{d}} u_{j} \frac{\partial}{\partial x_{j}}\left(\frac{\gamma-b \rho}{\gamma-1} \mathcal{B}\right) & =0,
\end{aligned}
$$

respectively. We emphasize that in order to have the correct pressure equilibrium in (10), these are the two key 
equations that should be satisfied and approximated consistently (when the problem is solved numerically, see Section 3). On the other hand, as a practical matter, it is obvious that, in addition to (11) and (12), we need to impose one more additional condition so as to have enough equations for the determination of all the three material quantities in (8). In our approach, see [33], this is done by simply breaking (11) into the following two parts:

$$
\begin{aligned}
& \frac{\partial}{\partial t}\left(\frac{1}{\gamma-1}\right)+\sum_{j=1}^{N_{d}} u_{j} \frac{\partial}{\partial x_{j}}\left(\frac{1}{\gamma-1}\right)=0 \\
& \frac{\partial}{\partial t}\left(\frac{b \rho}{\gamma-1}\right)+\sum_{j=1}^{N_{d}} u_{j} \frac{\partial}{\partial x_{j}}\left(\frac{b \rho}{\gamma-1}\right)=0 .
\end{aligned}
$$

Having done so, we arrive at a system of three equations (12), (13), and (14) for the variables $(\gamma-b \rho) \mathcal{B} /(\gamma-1)$, $1 /(\gamma-1)$, and $b \rho /(\gamma-1)$, in the order mentioned. Combining them to (2) yields a model system that is fundamental in our algorithm for describing the behavior of the numerical mixing between the barotropic and non-barotropic components near the interface. With that, there is no difficulty to compute the pressure based on the equation of state,

$$
p=\left[\rho E-\frac{\sum_{j=1}^{N_{d}}\left(\rho u_{j}\right)^{2}}{2 \rho}-\frac{\gamma-b \rho}{\gamma-1} \mathcal{B}\right] /\left(\frac{1-b \rho}{\gamma-1}\right) .
$$

Up to this point, our discussion stresses only on an approach that is capable of keeping the pressure in equilibrium for a model interface-only problem. Since in practice we are interested in shock wave problems as well, we should take the equations, i.e., (12), (13), and (14), in a form such that $\gamma, b$, and $\mathcal{B}$ remain unchanged across both shocks and rarefaction waves. In this regard, it is known that with $\gamma$ governed by (13), there is no problem to do so (cf. $[1,32]$ ). For $b$ and $\mathcal{B}$, however, due to the appearance of the density term in (12) and (14), it turns out that, in a time when such a situation occurs, for consistent with the mass conservation law of the fluid mixture they should be modified by

$$
\begin{array}{r}
\frac{\partial}{\partial t}\left(\frac{\gamma-b \rho}{\gamma-1} \mathcal{B}\right)+\sum_{j=1}^{N_{d}} \frac{\partial}{\partial x_{j}}\left(\frac{\gamma-b \rho}{\gamma-1} \mathcal{B} u_{j}\right)= \\
\frac{\gamma \mathcal{B}}{\gamma-1} \sum_{j=1}^{N_{d}} \frac{\partial u_{j}}{\partial x_{j}}
\end{array}
$$

and

$$
\frac{\partial}{\partial t}\left(\frac{b \rho}{\gamma-1}\right)+\sum_{j=1}^{N_{d}} \frac{\partial}{\partial x_{j}}\left(\frac{b \rho}{\gamma-1} u_{j}\right)=0
$$

respectively, so that the mass-conserving property of the solution in the single component region can be acquired also (cf. [33]). We note that, for convenience, we call the set of equations: (13), (15), and (16), a $\gamma$-based effective equations for the mixture of the material quantities of the modified Noble-Abel equation of state to be distinct from the other one presented below.

\subsection{2 $\alpha$-based effective equations}

Before proceeding further, we comment that to find the initial fluid mixtures, $1 /(\gamma-1),(\gamma-b \rho) \mathcal{B} /(\gamma-1)$, and $b \rho /(\gamma-1)$, that is necessary when we initialize the data for multicomponent flow computations, we use the equation of state (8), where written as a function of the volume fraction $\alpha_{\iota}$, for $\iota=1,2$, and $\alpha_{1}+\alpha_{2}=1$, it reads

$$
\begin{aligned}
\frac{1-b \rho}{\gamma-1} p+\frac{\gamma-b \rho}{\gamma-1} \mathcal{B} & =\rho e=\sum_{\iota=1}^{2} \alpha_{\iota} \rho_{\iota} e_{\iota} \\
& =\sum_{\iota=1}^{2} \alpha_{\iota}\left(\frac{1-b_{\iota} \rho_{\iota}}{\gamma_{\iota}-1} p_{\iota}+\frac{\gamma_{\iota}-b_{\iota} \rho_{\iota}}{\gamma_{\iota}-1} \mathcal{B}_{\iota}\right) .
\end{aligned}
$$

Here the subscript " $\iota$ " denotes the state variable of fluid component $\iota$. By taking a similar approach as employed in Section 2.2.1 for the derivation of the $\gamma$-based effective equation it comes out readily a splitting of the above expression into the relations:

$$
\begin{aligned}
\frac{1}{\gamma-1} & =\sum_{\iota=1}^{2} \frac{\alpha_{\iota}}{\gamma_{\iota}-1}, \frac{\gamma-b \rho}{\gamma-1} \mathcal{B}=\sum_{\iota=1}^{2} \alpha_{\iota} \frac{\gamma_{\iota}-b_{\iota} \rho_{\iota}}{\gamma_{\iota}-1} \mathcal{B}_{\iota} \\
\frac{b \rho}{\gamma-1} & =\sum_{\iota=1}^{2} \alpha_{\iota} \frac{b_{\iota} \rho_{\iota}}{\gamma_{\iota}-1}
\end{aligned}
$$

where in the process of splitting the terms the pressure $p$ is chosen to fulfill the condition

$$
\left(\frac{1-b \rho}{\gamma-1}\right) p=\sum_{\iota=1}^{2} \alpha_{\iota}\left(\frac{1-b_{\iota} \rho_{\iota}}{\gamma_{\iota}-1}\right) p_{\iota} .
$$

Notice that when each of the partial pressures is in an equilibrium state within a grid cell, in conjunction with the first and third parts of (17), the pressure $p$ obtained from (18) would remain in the same equilibrium as well, i.e., $p=p_{\iota}$, for $\iota=1,2$.

Now if the above volume-fraction notion of the states $1 /(\gamma-1),(\gamma-b \rho) \mathcal{B} /(\gamma-1)$, and $b \rho /(\gamma-1)$ are being employed in the $\gamma$-based effective equations, we are able to rewrite them straightforwardly into a componentwise form as

$$
\begin{gathered}
\frac{\partial}{\partial t}\left(\frac{\alpha_{\iota}}{\gamma_{\iota}-1}\right)+\sum_{j=1}^{N_{d}} u_{j} \frac{\partial}{\partial x_{j}}\left(\frac{\alpha_{\iota}}{\gamma_{\iota}-1}\right)=0 \\
\frac{\partial}{\partial t}\left(\alpha_{\iota} \frac{\gamma_{\iota}-b_{\iota} \rho_{\iota}}{\gamma_{\iota}-1} \mathcal{B}_{\iota}\right)+\sum_{j=1}^{N_{d}} \frac{\partial}{\partial x_{j}}\left(\alpha_{\iota} \frac{\gamma_{\iota}-b_{\iota} \rho_{\iota}}{\gamma_{\iota}-1} \mathcal{B}_{\iota} u_{j}\right) \\
=\alpha_{\iota} \frac{\gamma_{\iota} \mathcal{B}_{\iota}}{\gamma_{\iota}-1} \sum_{j=1}^{N_{d}} \frac{\partial u_{j}}{\partial x_{j}} \\
\frac{\partial}{\partial t}\left(\alpha_{\iota} \frac{b_{\iota} \rho_{\iota}}{\gamma_{\iota}-1}\right)+\sum_{j=1}^{N_{d}} \frac{\partial}{\partial x_{j}}\left(\alpha_{\iota} \frac{b_{\iota} \rho_{\iota}}{\gamma_{\iota}-1} u_{j}\right)=0
\end{gathered}
$$

for $\iota=1,2$. Then based on the fact that all the material quantities $\gamma_{\iota}, b_{\iota}$, and $\mathcal{B}_{\iota}$ will be kept as a constant in each 
phase of the domain at all time, from (19), it is easy to find the transport equation for the volume fraction $\alpha_{\iota}$ as

$$
\frac{\partial \alpha_{\iota}}{\partial t}+\sum_{j=1}^{N_{d}} u_{j} \frac{\partial \alpha_{\iota}}{\partial x_{j}}=0
$$

whereas from (21), we find the mass conservation law for the fluid component $\iota$ as

$$
\frac{\partial}{\partial t}\left(\rho_{\iota} \alpha_{\iota}\right)+\sum_{j=1}^{N_{d}} \frac{\partial}{\partial x_{j}}\left(\rho_{\iota} \alpha_{\iota} u_{j}\right)=0 .
$$

Furthermore, in the case of (20), after some simple algebraic manipulations, it can be expressed by the form

$$
\begin{aligned}
\frac{\gamma_{\iota} \mathcal{B}_{\iota}}{\gamma_{\iota}-1}\left[\frac{\partial \alpha_{\iota}}{\partial t}+\sum_{j=1}^{N_{d}} u_{j} \frac{\partial \alpha_{\iota}}{\partial x_{j}}\right]- & \frac{b_{\iota} \mathcal{B}_{\iota}}{\gamma_{\iota}-1}\left[\frac{\partial}{\partial t}\left(\rho_{\iota} \alpha_{\iota}\right)+\right. \\
& \left.\sum_{j=1}^{N_{d}} \frac{\partial}{\partial x_{j}}\left(\rho_{\iota} \alpha_{\iota} u_{j}\right)\right]=0
\end{aligned}
$$

yielding naturally the same set of governing equations as before, i.e., (22) and (23). It is apparent that, if the solutions of $\alpha_{\iota}$ and $\rho_{\iota} \alpha_{\iota}$ are known from the equations, we may therefore compute $1 /(\gamma-1),(\gamma-b \rho) \mathcal{B} /(\gamma-1)$, and $b \rho /(\gamma-1)$ directly according to (17). Thus, instead of using the $\gamma$-based effective equations, it is a viable alternate to use the $\alpha$-based equations: (22) and (23), for the motion of the mixture of the material quantities of the problem.

\subsubsection{Complete model system}

It is important to mention that to make up a complete model system that is capable of dealing with all the fluid component cases, $\alpha=1$ (barotropic phase), $\alpha=0$ (non-barotropic phase), or $\alpha \in(0,1)$ (barotropic and non-barotropic coexistence phase), we have to know the approximate location of the interfaces so that the correct equations of motion as well as the equation of state can be employed to each part of the domain, from the current time to the next. Here, since $\alpha_{1}+\alpha_{2}=1$, it is clear that if we choose $\alpha_{1}=\alpha$ and so $\alpha_{2}=1-\alpha$, the two transport equations in (22) for each of $\alpha_{1}$ and $\alpha_{2}$ can be combined, without affecting anything, to a single one for $\alpha$ as

$$
\frac{\partial \alpha}{\partial t}+\sum_{j=1}^{N_{d}} u_{j} \frac{\partial \alpha}{\partial x_{j}}=0
$$

leading to the evolution equation we use in the algorithm for that matter. Note that, in devising a fluid-mixture type algorithm for multicomponent problems, one common practice is to consider the mixture of the total density, $\rho=\rho_{1} \alpha_{1}+\rho_{2} \alpha_{2}$, as one of the basic variables in the proposed model system (cf. $[32,33,35])$. When this is the case, it should be more sensible to include only one of the equations in (23) together with the last two relations in (17) than the use of both (15) and (16) for the variables $(\gamma-b \rho) \mathcal{B} /(\gamma-1)$ and $b \rho /(\gamma-1)$.

Putting all the things together, with the equation of state (9), the model equations with which we propose to solve the present two-fluid flow problems in more than one space dimension take the form

$$
\begin{aligned}
\frac{\partial \rho}{\partial t}+\sum_{j=1}^{N_{d}} \frac{\partial}{\partial x_{j}}\left(\rho u_{j}\right) & =0 \\
\frac{\partial}{\partial t}\left(\rho u_{i}\right)+\sum_{j=1}^{N_{d}} \frac{\partial}{\partial x_{j}}\left(\rho u_{i} u_{j}+p \delta_{i j}\right) & =0 \\
\frac{\partial}{\partial t}(\rho E)+\sum_{j=1}^{N_{d}} \frac{\partial}{\partial x_{j}}\left(\rho E u_{j}+p u_{j}\right) & =0 \quad \text { if } \alpha \neq 1 \\
\frac{\partial}{\partial t}\left(\rho_{1} \alpha\right)+\sum_{j=1}^{N_{d}} \frac{\partial}{\partial x_{j}}\left(\rho_{1} \alpha u_{j}\right) & =0 \\
\frac{\partial \alpha}{\partial t}+\sum_{j=1}^{N_{d}} u_{j} \frac{\partial \alpha}{\partial x_{j}} & =0,
\end{aligned}
$$

for $i=1,2, \cdots, N_{d}$. Note that when $\alpha \neq 1$ we have a system of $N_{d}+4$ equations for the motion of both the pure non-barotropic phase and the fluid mixture. Clearly, the first $N_{d}+2$ of them are simply the basic conservation of the mass, momenta $\left(N_{d}\right.$ of them), and total energy, while the last two equations are (23) and (24) that are used to find the variables $1 /(\gamma-1),(\gamma-b \rho) \mathcal{B} /(\gamma-1)$, and $b \rho /(\gamma-1)$ from the relations in (17), and also the approximate location of the interface (the volume fraction function $\alpha$ does it). On the other instance, when $\alpha=1$, we just have the first $N_{d}+1$ equations governing the single-component barotropic flow as usual. With a system expressing in this way, there is no problem to compute all the state variables of interest, including the pressure from the equation of state. The initialization of the state variables in (25) for fluid-mixture cells can be made in a standard way as described in $[32,33]$ for numerical simulations.

It should be remarked that for an easy extension of the proposed model to more complex equations of state we have taken (25) in a form that is analogous to the five-equation model advocated by Allaire et al. [3] or Massoni et al. [24] for compressible multicomponent flow problems with general non-barotropic equation of state. For any given $N_{d}$, if the state variables of the flow are all in the region of the thermodynamic stability (this is the case we are interested in here), it is not difficult to show that (25) is a hyperbolic system in the sense that any linear combination of the matrices $A_{j}, j=1,2, \cdots, N_{d}$, appearing in the quasi-linear form of the equations

$$
\frac{\partial q}{\partial t}+\sum_{j=1}^{N_{d}} A_{j}(q) \frac{\partial q}{\partial x_{j}}=0
$$

has real eigenvalues and a complete set of eigenvectors.

As an example, we consider the three-dimensional case $N_{d}=3$, and then have the state vector $q$ in (26) defined 
by

$$
q=\left(\rho, \rho u_{1}, \rho u_{2}, \rho u_{3}, \rho E, \rho_{1} \alpha, \alpha\right)^{T},
$$

and the matrices $A_{j}$, for $j=1,2,3$, defined by

$A_{1}=$

$\left[\begin{array}{ccccccc}0 & 1 & 0 & 0 & 0 & 0 & 0 \\ K-u_{1}^{2} & 2 u_{1}+\eta_{2} & \eta_{3} & \eta_{4} & \Gamma & \varphi & \chi \\ -u_{1} u_{2} & u_{2} & u_{1} & 0 & 0 & 0 & 0 \\ -u_{1} u_{3} & u_{3} & 0 & u_{1} & 0 & 0 & 0 \\ u_{1}(K-H) & H+u_{1} \eta_{2} & u_{1} \eta_{3} & u_{1} \eta_{4} & u_{1}(\Gamma+1) & u_{1} \varphi & u_{1} \chi \\ -u_{1} \rho_{1} \alpha / \rho & \rho_{1} \alpha / \rho & 0 & 0 & 0 & u_{1} & 0 \\ 0 & 0 & 0 & 0 & 0 & 0 & u_{1}\end{array}\right]$

$A_{2}=$

$\left[\begin{array}{ccccccc}0 & 0 & 1 & 0 & 0 & 0 & 0 \\ -u_{1} u_{2} & u_{2} & u_{1} & 0 & 0 & 0 & 0 \\ K-u_{2}^{2} & \eta_{2} & 2 u_{2}+\eta_{3} & \eta 4 & \Gamma & \varphi & \chi \\ -u_{2} u_{3} & 0 & u_{3} & u_{2} & 0 & 0 & 0 \\ u_{2}(K-H) & u_{2} \eta_{2} & H+u_{2} \eta_{3} & u_{2} \eta_{4} & u_{2}(\Gamma+1) & u_{2} \varphi & u_{2} \chi \\ -u_{2} \rho_{1} \alpha / \rho & 0 & \rho_{1} \alpha / \rho & 0 & 0 & u_{2} & 0 \\ 0 & 0 & 0 & 0 & 0 & 0 & u_{2}\end{array}\right]$

$A_{3}=$

$\left[\begin{array}{ccccccc}0 & 0 & 0 & 1 & 0 & 0 & 0 \\ -u_{1} u_{3} & u_{3} & 0 & u_{1} & 0 & 0 & 0 \\ -u_{2} u_{3} & 0 & u_{3} & u_{2} & 0 & 0 & 0 \\ K-u_{3}^{2} & \eta_{2} & \eta_{3} & 2 u_{3}+\eta_{4} & \Gamma & \varphi & \chi \\ u_{3}(K-H) & u_{3} \eta_{2} & u_{3} \eta_{3} & H+u_{3} \eta_{4} & u_{3}(\Gamma+1) & u_{3} \varphi & u_{3} \chi \\ -u_{3} \rho_{1} \alpha / \rho & 0 & 0 & \rho_{1} \alpha / \rho & 0 & u_{3} & 0 \\ 0 & 0 & 0 & 0 & 0 & 0 & u_{3}\end{array}\right]$.

With that, the eigenvalues and the corresponding eigenvectors of the matrices are:

for matrix $A_{1}$,

$$
\begin{aligned}
\Lambda_{A_{1}}= & \operatorname{diag}\left(\lambda_{1}^{(1)}, \lambda_{2}^{(1)}, \cdots, \lambda_{7}^{(1)}\right) \\
= & \operatorname{diag}\left(u_{1}-c, u_{1}, u_{1}+c, u_{1}, \cdots, u_{1}\right), \\
R_{A_{1}}= & \left(r_{1}^{(1)}, r_{2}^{(1)}, \cdots, r_{7}^{(1)}\right) \\
& {\left[\begin{array}{ccccccc}
1 & 1 & 1 & 0 & 0 & 0 & 0 \\
u_{1}-c & u_{1} & u_{1}+c & 0 & 0 & 0 & 0 \\
u_{2} & u_{2} & u_{2} & 1 & 0 & 0 & 0 \\
u_{3} & u_{3} & u_{3} & 0 & 1 & 0 & 0 \\
H-u_{1} c & K / \Gamma & H+u_{1} c & u_{2} & u_{3} & -\varphi / \Gamma & -\chi / \Gamma \\
\rho_{1} \alpha / \rho & 0 & \rho_{1} \alpha / \rho & 0 & 0 & 1 & 0 \\
0 & 0 & 0 & 0 & 0 & 0 & 1
\end{array}\right], }
\end{aligned}
$$

for matrix $A_{2}$,

$$
\begin{aligned}
\Lambda_{A_{2}}= & \operatorname{diag}\left(\lambda_{1}^{(2)}, \lambda_{2}^{(2)}, \cdots, \lambda_{7}^{(2)}\right) \\
= & \operatorname{diag}\left(u_{2}-c, u_{2}, u_{2}+c, u_{2}, \cdots, u_{2}\right), \\
R_{A_{2}}= & \left(r_{1}^{(2)}, r_{2}^{(2)}, \cdots, r_{7}^{(2)}\right) \\
& {\left[\begin{array}{ccccccc}
1 & 1 & 1 & 0 & 0 & 0 & 0 \\
u_{1} & u_{1} & u_{1} & 1 & 0 & 0 & 0 \\
u_{2}-c & u_{2} & u_{2}+c & 0 & 0 & 0 & 0 \\
u_{3} & u_{3} & u_{3} & 0 & 1 & 0 & 0 \\
H-u_{2} c & K / \Gamma & H+u_{2} c & u_{1} & u_{3} & -\varphi / \Gamma & -\chi / \Gamma \\
\rho_{1} \alpha / \rho & 0 & \rho_{1} \alpha / \rho & 0 & 0 & 1 & 0 \\
0 & 0 & 0 & 0 & 0 & 0 & 1
\end{array}\right], }
\end{aligned}
$$

and for matrix $A_{3}$,

$$
\begin{aligned}
\Lambda_{A_{3}}= & \operatorname{diag}\left(\lambda_{1}^{(3)}, \lambda_{2}^{(3)}, \cdots, \lambda_{7}^{(3)}\right) \\
= & \operatorname{diag}\left(u_{3}-c, u_{3}, u_{3}+c, u_{3}, \cdots, u_{3}\right), \\
R_{A_{3}}= & \left(r_{1}^{(3)}, r_{2}^{(3)}, \cdots, r_{7}^{(3)}\right) \\
& {\left[\begin{array}{ccccccc}
1 & 1 & 1 & 0 & 0 & 0 & 0 \\
u_{1} & u_{1} & u_{1} & 1 & 0 & 0 & 0 \\
u_{2} & u_{2} & u_{2} & 0 & 1 & 0 & 0 \\
u_{3}-c & u_{3} & u_{3}+c & 0 & 0 & 0 & 0 \\
H-u_{3} c & K / \Gamma & H+u_{3} c & u_{1} & u_{2} & -\varphi / \Gamma & -\chi / \Gamma \\
\rho_{1} \alpha / \rho & 0 & \rho_{1} \alpha / \rho & 0 & 0 & 1 & 0 \\
0 & 0 & 0 & 0 & 0 & 0 & 1
\end{array}\right] ; }
\end{aligned}
$$

$A_{j} r_{k}^{(j)}=\lambda_{k}^{(j)} r_{k}^{(j)}, j=1,2,3$, and $k=1,2, \cdots, 7$. Here $c=\sqrt{[\gamma /(1-b \rho)][(p+\mathcal{B}) / \rho]}$ is the speed of sound of the fluid, and the other notations appeared in the above formulae are set by $\Gamma=(\gamma-1) /(1-b \rho)$, $K=\left(\Gamma \sum_{j=1}^{3} u_{j}^{2} / 2\right)+\zeta, H=E+p / \rho, \eta_{i}=-u_{i} \Gamma$, for $i=1,2,3, \varphi=\Gamma \Delta[b \mathcal{B} /(\gamma-1)]-p \Delta[b /(\gamma-1)] / \Gamma$, and $\chi=-\Gamma \Delta[\gamma \mathcal{B} /(\gamma-1)]+p \Delta[1 /(\gamma-1)] / \Gamma$, where $\zeta=-b_{2} /\left(\gamma_{2}-1\right)\left[\left(\Gamma \mathcal{B}_{2}\right)+(p / \Gamma)\right]$ and $\Delta[\kappa]=\kappa_{1}-\kappa_{2}$.

It should be noted that since the volume fraction function $\alpha$ is governed by a linear transport equation of the form (24) and its solution can only have jumps across the material interfaces, but not across other waves such as shock and rarefaction, we find easily the usual expression of the Rankine-Hugoniot jump conditions across shock waves for this two-fluid model (25) (cf. [33]). Finally, for the ease of the latter reference, it is customary to write (25) into a more compact expression by

$$
\frac{\partial q}{\partial t}+\sum_{j=1}^{N_{d}} f_{j}\left(\frac{\partial}{\partial x_{j}}, q\right)=0
$$

where $f_{j}$ is taken as the vector-value function of the form

$$
\begin{gathered}
f_{j}=\left[\frac{\partial}{\partial x_{j}}\left(\rho u_{j}\right), \frac{\partial}{\partial x_{j}}\left(\rho u_{1} u_{j}+p \delta_{1 j}\right), \cdots, \frac{\partial}{\partial x_{j}}\left(\rho u_{N_{d}} u_{j}\right.\right. \\
\left.\left.+p \delta_{N_{d} j}\right), \frac{\partial}{\partial x_{j}}\left(\rho E u_{j}+p u_{j}\right), \frac{\partial}{\partial x_{j}}\left(\rho_{1} \alpha u_{j}\right), u_{j} \frac{\partial \alpha}{\partial x_{j}}\right]^{T},
\end{gathered}
$$

for $j=1,2, \cdots, N_{d}$.

\section{Numerical approximations}

To find approximate solutions of our two-fluid model presented in Section 2, we use a standard high-resolution wave propagation method developed by LeVeque $[17,18]$ for general hyperbolic systems of partial differential equations. This method is a different form of the fluctuationand-signal scheme of Roe $[29,30]$ in that we solve onedimensional Riemann problems at each cell interface, and use the resulting waves (i.e., discontinuities moving at constant speeds) to update the solutions in neighboring grid 
cells. To achieve second-order accurate on smooth solutions, and sharp and monotone profiles on discontinuous solutions, we introduce slopes and limiters to the method as in many other high-resolution schemes for conservation laws $[11,19,39]$.

\subsection{HLL-type approximate Riemann solvers}

As a preliminary, we begin by reviewing the construction of the approximate Riemann problem solutions in one space dimension which is one of the major elements in our numerical algorithm. If we consider the case $N_{d}=3$ as an example, to simplified the notation, rather than using $x_{j}$ for the spatial variable and $u_{j}$ for the particle velocity, for $j=1,2,3$, we take the often-employed symbols $(x, y, z)$ and $(u, v, w)$ for that matter instead. Then the problem to be solved is to find the solution of (25) in the direction normal to one of the $x y, y z$, and $x z$ planes, with piecewise constant data $q_{L}$ and $q_{R}$ to the left and right of the cell interface. It should be noted that in the applications concerned here, we have chosen the data $q_{L}$ and $q_{R}$ well enough so that the solution of the Riemann problem would consist of genuinely nonlinear waves such as shock and rarefaction, and linearly degenerate wave such as contact discontinuity; there is no vacuum region occurring in the solution. Without loss of generality, in the following we only look at the Riemann problem in the direction normal to the $y z$ plane.

There are a wide variety of approaches proposed in the literature for determining an approximate solution of the Riemann problem, see [41], for example. Here we are interested in a simple variant of the Riemann solver based on the work of Harten, Lax, and van Leer [13] for hyperbolic systems of conservation laws,

$$
\frac{\partial q}{\partial t}+\frac{\partial}{\partial x} f(q)=0
$$

where $q \in \mathbb{R}^{n}$ is the vector of conserved variables for a system of $n$ equations, and $f$ is the flux function. Recall that in the original version of the HLL solver, the solution of the Riemann problem is assumed to be composed of two discontinuities propagating at constant speeds $\lambda_{L}$ and $\lambda_{R}$ to the left and right, $\lambda_{L} \neq \lambda_{R}$, separating three constant states in the space-time domain. If we assume further that $\lambda_{L}$ and $\lambda_{R}$ are known a priori by some simple estimates based on the local information of the wave speeds, then by using the integral form of the conservation laws over a sufficiently large control volume $[-M, M] \times[0, T]$, for some positive $M$ and $T \in \mathbb{R}$, it is easy to find the constant state in the middle region, denoted by $q_{m}$, as the average of the exact solution over the interval $\left[\lambda_{L} T, \lambda_{R} T\right]$ at time $T$,

$$
\begin{aligned}
q_{m} & =\frac{1}{\left(\lambda_{R}-\lambda_{L}\right) T} \int_{\lambda_{L} T}^{\lambda_{R} T} q(x, T) d x \\
& =\frac{\lambda_{R} q_{R}-\lambda_{L} q_{L}-f\left(q_{R}\right)+f\left(q_{L}\right)}{\lambda_{R}-\lambda_{L}},
\end{aligned}
$$

where $f\left(q_{\iota}\right)$ is the flux evaluated at the state $q_{\iota}$, for $\iota=L$ or $R$.
In spite of the fact that the above 2-wave HLL solver has been applied quite successfully to many numerical methods for solving problems governed by (28), it is known however that the numerical result obtained by using this solver is too diffusive for contact discontinuities. In addition to that, because of the lack of information on the structure of the material interfaces, it is not feasible at all to the more general multicomponent problems like the one considered in this article. Nevertheless, as recommended by Toro et al. [6,42], this 2 -wave Riemann solver can be improved quite easily by introducing an additional middle wave of speed $u_{m}$ in the solution structure for modeling the speed of contact discontinuity, yielding a 3 -wave HLL (or called HLLC) solver. Note that if we have had the first two components of $q_{m}$ computed from (29), we may simply set $u_{m}=q_{m}^{(2)} / q_{m}^{(1)}$ (cf. $[31,36]$ ), where $q_{m}^{(i)}$ is the $i$ th component of the vector $q_{m}$, see [41] for the various other possible choices.

With that, our goal next is to find the constant states $q_{m L}$ and $q_{m R}$ in the regions $m_{L}$ and $m_{R}$ to the left and right of the middle wave, respectively. We do this by making use of the integral form of the conservation laws again, but now applied over a control volume $\left[-M, u_{m} T-\epsilon\right] \times$ $[0, T]$ for the state $q_{m L}$, and over a control volume $\left[u_{m} T+\right.$ $\epsilon, M] \times[0, T]$ for the state $q_{m R}, 0<\epsilon \ll 1$. When the aforementioned procedure is taken effect to the current two-fluid model (25) in the prescribed direction, it is not difficult to show that the result is

$$
\begin{array}{r}
q_{m \iota}^{(1)}=\frac{f_{m \iota}^{(1)}}{\lambda_{\iota}-u_{m}}, \quad q_{m \iota}^{(2)}=u_{m} q_{m \iota}^{(1)}, \quad q_{m \iota}^{(3)}=v_{\iota} q_{m \iota}^{(1)}, \\
q_{m \iota}^{(4)}=w_{\iota} q_{m \iota}^{(1)}, \quad q_{m \iota}^{(5)}=\frac{f_{m \iota}^{(5)}+u_{m}\left(u_{m} f_{m \iota}^{(1)}-f_{m \iota}^{(2)}\right)}{\lambda_{\iota}-u_{m}} \\
q_{m \iota}^{(6)}=\frac{f_{m \iota}^{(6)}}{\lambda_{\iota}-u_{m}}, \quad q_{m \iota}^{(7)}=q_{\iota}^{(7)},
\end{array}
$$

where $f_{m \iota}^{(i)}=\lambda_{\iota} q_{\iota}^{(i)}-f^{(i)}\left(q_{\iota}\right)$ represents the $i$ th component of the vector $f_{m \iota}$, for $\iota=L$ or $R$. It is interesting to remark that $q_{m L}^{(i)}$ and $q_{m R}^{(i)}$ satisfy the basic consistency condition of the integral form of the conservation laws,

$$
\left(\frac{u_{m}-\lambda_{L}}{\lambda_{R}-\lambda_{L}}\right) q_{m L}^{(i)}+\left(\frac{\lambda_{R}-u_{m}}{\lambda_{R}-\lambda_{L}}\right) q_{m R}^{(i)}=q_{m}^{(i)}
$$

for $i=1,2, \cdots, 6$. By taking $\lambda_{L}=\min \left(u_{L}-c_{L}, u_{R}-c_{R}\right)$ and $\lambda_{R}=\max \left(u_{L}+c_{L}, u_{R}+c_{R}\right)$, for instance, we may then set the speed of the three moving discontinuities by $\lambda_{1}=\lambda_{L}, \lambda_{2}=u_{m}, \lambda_{3}=\lambda_{R}$, and the jumps across each of them by

$\mathcal{W}_{1}=q_{m L}-q_{L}, \quad \mathcal{W}_{2}=q_{m R}-q_{m L}, \quad \mathcal{W}_{3}=q_{R}-q_{m R}$

using the result (30). As usual, wave propagation methods (to be described below) are based on using these propagating discontinuity to update the cell averages in the cells neighboring each interface. 


\subsection{Wave propagation methods}

\subsubsection{One-dimensional case}

To review the basic idea of our underlying integration scheme, it is instructive to look at the simplest $N_{d}=1$ case of our model system,

$$
\frac{\partial q}{\partial t}+f_{1}\left(\frac{\partial}{\partial x}, q\right)=0
$$

with $q$ and $f_{1}$ defined as in (25) and (27). For simplicity, we describe the method on a uniform grid with fixed mesh spacing $\Delta x$ in the $x$-direction, and use a standard finitevolume formulation in which the discretized solution $Q_{j}^{n}$ approximates the cell average over the grid cell $\left[x_{j}, x_{j+1}\right]$ at time $t_{n}$

$$
Q_{j}^{n} \approx \frac{1}{\Delta x} \int_{x_{j}}^{x_{j+1}} q\left(x, t_{n}\right) d x
$$

The time step from the current time $t_{n}$ to the next $t_{n+1}$ is denoted by $\Delta t$.

In this numerical setup, a first order accurate version of the method in wave-propagation form is a Godunov-type scheme that can be written as

$$
Q_{j}^{n+1}=Q_{j}^{n}-\frac{\Delta t}{\Delta x} \sum_{m=1}^{m_{w}}\left(\lambda_{m}^{-} \mathcal{W}_{m}\right)_{j+1}^{n}+\left(\lambda_{m}^{+} \mathcal{W}_{m}\right)_{j}^{n}
$$

Here $\lambda_{m}$ and $\mathcal{W}_{m}$ are solutions of the $m$ th wave family, for $m=1,2, \cdots, m_{w}$, obtained from solving the Riemann problems at cell interfaces $x_{j}$ and $x_{j+1}$ with a properly chosen solver, and $\lambda^{-}=\min (\lambda, 0), \lambda^{+}=\max (\lambda, 0)$. It is known that method (31) belongs to a class of upwind schemes, and is stable when the typical CFL (CourantFriedrichs-Lewy) condition is satisfied (cf. $[11,19])$. Moreover, it is not difficult to show that the method is quasiconservative in the sense that when applying the method to (25) not only the conservation laws but also the transport equation is approximated in a consistent manner by the method, see [33] for the details.

To improve the accuracy of (31) to high resolution, it is a standard approach by first introducing correction waves in a piecewise-linear form with zero mean value, and then propagating each wave over the time step $\Delta t$ to update the cell averages it overlaps. Without providing the details in this place (cf. [19] for example), with the corrections, (31) is modified by

$$
\begin{array}{r}
Q_{j}^{n+1}:=Q_{j}^{n+1}-\frac{1}{2} \sum_{m=1}^{m_{w}}\left(\left|\mu_{m}\right|\left(1-\left|\mu_{m}\right|\right) \widetilde{\mathcal{W}}_{m}\right)_{j+1}^{n}- \\
\left(\left|\mu_{m}\right|\left(1-\left|\mu_{m}\right|\right) \widetilde{\mathcal{W}}_{m}\right)_{j}^{n}
\end{array}
$$

where $\mu_{m}=\lambda_{m} \Delta t / \Delta x$, and $\widetilde{\mathcal{W}}_{m}$ is a limited value of $\mathcal{W}_{m}$ obtained by comparing $\mathcal{W}_{m}$ with the corresponding $\mathcal{W}_{m}$ from the neighboring Riemann problem to the left (if $\lambda_{m}>0$ ) or to the right (if $\lambda_{m}<0$ ).
Note that when utilizing the wave-form representation of the Riemann problem solution, it is a common practice to perform the limiting procedure over each component of the wave via a limiter function $\Phi$ (e.g., by using the minmod function $\Phi(\theta)=\max (0, \min (1, \theta))$ or some others as discussed in [39]), and set

$$
\begin{gathered}
\widetilde{\mathcal{W}}_{m j}^{(i)}=\Phi\left(\theta_{m j}^{(i)}\right) \mathcal{W}_{m j}^{(i)} \quad \text { with } \quad \theta_{m j}^{(i)}=\frac{\mathcal{W}_{m J}^{(i)}}{\mathcal{W}_{m j}^{(i)},} \\
J=\left\{\begin{array}{lll}
j-1 & \text { if } & \lambda_{m j} \geq 0 \\
j+1 & \text { if } & \lambda_{m j}<0
\end{array},\right.
\end{gathered}
$$

where $\mathcal{W}_{m j}^{(i)}$ is the $i$ th component of $\mathcal{W}_{m j}$. While the above approach works in a satisfactory manner for many single component problems, it was shown in [36] that, for general multicomponent problems, to ensure a consistent approximation of the total energy near the interfaces the third limited component of the 2 -wave, $\widetilde{\mathcal{W}}_{2 j}^{(3)}$, should be modified so that the desired pressure equilibrium can be maintained there.

Having this in mind, following the previous work done in [36], we make a new definition of $\widetilde{\mathcal{W}}_{2 j}^{(3)}$ as

$$
\begin{array}{r}
\widetilde{\mathcal{W}}_{2 j}^{(3)}:=\Phi\left(\frac{\overline{\mathcal{W}}_{2 J}}{\overline{\mathcal{W}}_{2 j}}\right) \overline{\mathcal{W}}_{2 j}-\Delta\left[\frac{b \mathcal{B}}{\gamma-1}\right] \widetilde{\mathcal{W}}_{2 j}^{(4)}+ \\
\Delta\left[\frac{\gamma \mathcal{B}}{\gamma-1}\right] \widetilde{\mathcal{W}}_{2 j}^{(5)},
\end{array}
$$

where $\overline{\mathcal{W}}_{2 \iota}=\mathcal{W}_{2 \iota}^{(3)}+\Delta[b \mathcal{B} /(\gamma-1)] \mathcal{W}_{2 \iota}^{(4)}-\Delta[\gamma \mathcal{B} /(\gamma-$ 1)] $\mathcal{W}_{2 \iota}^{(5)}$, for $\iota=j$ and $J$ (i.e., $j-1$ or $j+1$ depending on the propagating direction of $\left.\lambda_{2 j}\right) ; \Delta[\kappa]=\kappa_{1}-\kappa_{2}$. With this revision of the limited 2-wave on the total energy, it is not difficult to show that for a model interface-only problem (see Section 2.2) we again have the required pressure equilibrium without introducing any spurious oscillations in the approximate solution. Besides that we would typically obtain a better resolution of the result as compared to the first order result, see [36] for the details.

\subsubsection{Multidimensional case}

To extend the one-dimensional wave propagation method to more space dimensions, here we take a simple dimensional-splitting approach in which a multidimensional problem is split into a sequence of one-dimensional problems. Consider the three-dimensional case $N_{d}=3$, for example. The hybrid barotropic and non-barotropic two-fluid flow problem modeled by (27), i.e.,

$$
\frac{\partial q}{\partial t}+f_{1}\left(\frac{\partial}{\partial x}, q\right)+f_{2}\left(\frac{\partial}{\partial y}, q\right)+f_{3}\left(\frac{\partial}{\partial z}, q\right)=0
$$


can be split into

$$
\begin{array}{ll}
x \text {-sweeps: } & \frac{\partial q}{\partial t}+f_{1}\left(\frac{\partial}{\partial x}, q\right)=0 \\
y \text {-sweeps: } & \frac{\partial q}{\partial t}+f_{2}\left(\frac{\partial}{\partial y}, q\right)=0 \\
z \text {-sweeps: } & \frac{\partial q}{\partial t}+f_{3}\left(\frac{\partial}{\partial z}, q\right)=0 .
\end{array}
$$

Assuming a uniform Cartesian grid with fixed mesh spacing $\Delta x, \Delta y$, and $\Delta z$ in the $x^{-}, y$-, and $z$-direction, respectively. In a finite-volume formulation of the solution, the value $Q_{i j k}^{n}$ would approximate the cell average of the solution over the $(i, j, k)$ th grid cell at time $t_{n}$,

$$
Q_{i j k}^{n} \approx \frac{1}{\Delta x \Delta y \Delta z} \iiint_{\mathcal{C}_{i j k}} q\left(x, y, z, t_{n}\right) d x d y d z
$$

where $\mathcal{C}_{i j k}=\left[x_{i}, x_{i+1}\right] \times\left[y_{j}, y_{j+1}\right] \times\left[z_{k}, z_{k+1}\right]$ denotes the cubical region occupied by the grid cell $(i, j, k)$. Then a dimensional-splitting (or called Godunov-splitting) version of the first-order wave propagation method in three dimensions can be written as

$$
\begin{gathered}
Q_{i j k}^{*}=Q_{i j k}^{n}-\frac{\Delta t}{\Delta x} \sum_{m=1}^{m_{w}}\left(\lambda_{m}^{(1)-} \mathcal{W}_{m}^{(1)}\right)_{i+1, j k}^{n}+ \\
\left(\lambda_{m}^{(1)+} \mathcal{W}_{m}^{(1)}\right)_{i j k}^{n}, \\
Q_{i j k}^{* *}=Q_{i j k}^{*}-\frac{\Delta t}{\Delta y} \sum_{m=1}^{m_{w}}\left(\lambda_{m}^{(2)-} \mathcal{W}_{m}^{(2)}\right)_{i, j+1, k}^{*}+ \\
\left(\lambda_{m}^{(2)+} \mathcal{W}_{m}^{(2)}\right)_{i j k}^{*} \\
Q_{i j k}^{n+1}=Q_{i j k}^{* *}-\frac{\Delta t}{\Delta z} \sum_{m=1}^{m_{w}}\left(\lambda_{m}^{(3)-} \mathcal{W}_{m}^{(3)}\right)_{i j, k+1}^{* *}+ \\
\left(\lambda_{m}^{(3)+} \mathcal{W}_{m}^{(3)}\right)_{i j k}^{* *} .
\end{gathered}
$$

Note that in the $x$-sweeps we start with cell average $Q_{i j k}^{n}$ at time $t_{n}$ and solve (34a) along each row of cells $\mathcal{C}_{i j k}$ with $j$ and $k$ fixed, updating $Q_{i j k}^{n}$ to $Q_{i j k}^{*}$ by the use of (35a), where $\lambda_{m, i j k}^{(1)}$ and $\mathcal{W}_{m, i j k}^{(1)}$ are solutions of the $m$ th wave family obtained from solving the onedimensional Riemann problems in the direction normal to the cell interface between $\mathcal{C}_{i j k}$ and $\mathcal{C}_{i+1, j k}$ with $Q_{i j k}^{n}$ and $Q_{i+1, j k}^{n}$ as initial data. Consequently in the $y$-sweeps we can use the $Q_{i j k}^{*}$ values as data for solving (34b) along each column of cells $\mathcal{C}_{i j k}$ with $i$ and $k$ fixed, which gives us $Q_{i j k}^{* *}$ from $(35 \mathrm{~b})$. Finally, in the $z$-sweeps we use the $Q_{i j k}^{* *}$ values as data for solving (34c) along the other column of cells $\mathcal{C}_{i j k}$ with $i$ and $j$ fixed, yielding the solution of the next time step $Q_{i j k}^{n+1}$ from (35c). Clearly, in each one-dimensional sweep, one may apply the same high-resolution approach as before to improve numerical accuracy of this splitting method.

It is a known fact that, except for some simple problems, there will be generally splitting error of the method just described. But from numerical experiences it turns out that the splitting error is often no worse than the errors introduced by the numerical methods in each sweep, and hence as a practical matter it is typically not necessary to use a more accurate splitting approach such as the Strang splitting [38] to reduce the splitting error, see [19] for some discussion of why one might not want to use a higher order splitting method. In addition, we have also observed good results for many practical problems obtained using the present method as compared to the fully discrete wave propagation method (cf. [16-18]). For these reasons, we will use the one-dimensional highresolution wave propagation method together with the Godunov splitting for all the multidimensional tests done in the next section.

\section{Numerical results}

We now show some numerical results to validate our twofluid algorithm described in Section 3. In all the problems considered below, we carry out the test using the HLLC Riemann solver, the "minmod" limiter in a high-resolution method, and the Courant number 0.9.

EXAMPLE 4.1. Our first example concerns a model shock-contact interaction problem in one dimension that verifies convergence of our numerical solutions to the weak ones in a multicomponent case. Here the initial condition we use is composed of a stationary air-water interface at $x=0.6 \mathrm{~m}$ within a one meter length domain, and a rightward going Mach 1.94 shock wave at $x=0.5 \mathrm{~m}$ traveling from the left to right. The fluid on the right of the interface is an air with the state variables

$$
(\rho, p, \alpha)_{R}=\left(1.2 \mathrm{~kg} / \mathrm{m}^{3}, 10^{5} \mathrm{~Pa}, 0\right),
$$

and the material quantities $\gamma=1.4, b=2 \times 10^{-3} \mathrm{~m}^{3} / \mathrm{kg}$, while the fluid on the left of the interface, (i.e., on the middle and the preshock state), is a water with the state variables

$$
(\rho, p, \alpha)_{M}=\left(10^{3} \mathrm{~kg} / \mathrm{m}^{3}, 10^{5} \mathrm{~Pa}, 1\right) .
$$

and the material quantities $\gamma=7, \mathcal{B}=3 \times 10^{8} \mathrm{~Pa}, \rho_{0}=$ $10^{3} \mathrm{~kg} / \mathrm{m}^{3}, p_{0}=10^{5} \mathrm{~Pa}$. The state behind the shock is taken to be

$$
\begin{aligned}
&(\rho, u, p, \alpha)_{L}=( 1337.61 \mathrm{~kg} / \mathrm{m}^{3}, 710.525 \mathrm{~m} / \mathrm{s}, \\
&\left.2 \times 10^{9} \mathrm{~Pa}, 1\right)
\end{aligned}
$$

see the dashed line shown in Fig. 1 for illustration. We note that in this instance since the interface is accelerated by a shock wave coming from the heavy-fluid to the lightfluid region, it is known that the resulting wave pattern after the interaction would consist of a transmitted shock wave, an interface, and a reflected rarefaction wave.

A snap shot of the computed solutions for $\rho_{1} \alpha, \rho-\rho_{1} \alpha$, $u$, and $p$ are shown in Fig. 1 at time $t=200 \mu \mathrm{s}$, where we solve the problem using the high-resolution method 

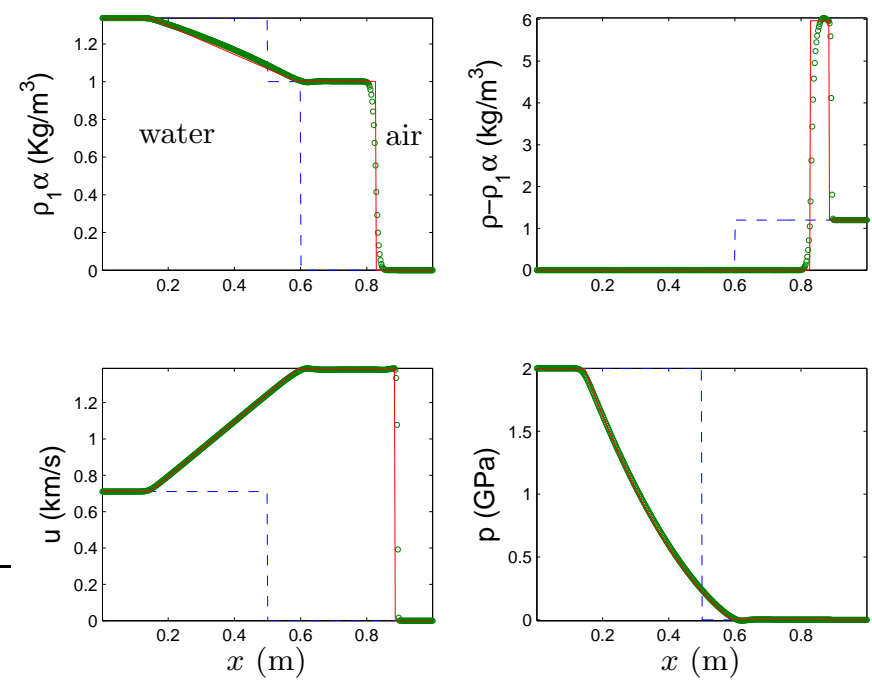

Fig. 1. High-resolution results for a model one-dimensional shock-contact interaction problem at time $t=200 \mu \mathrm{s}$. The solid line is the exact solution and the points show the computed solution with 400 mesh points. The dashed line in each subplot is the initial condition at time $t=0$.

with 400 mesh points. Observing the displayed profiles, we see clearly the good behavior of the computed air-water interface and also the shock and rarefaction waves as in comparison with the exact solution.

EXAMPLE 4.2. We are next concerned with a radially symmetric problem where the computed solutions in two space dimensions can be compared to the one-dimensional results for numerical validation. In this test, we use the following two-phase (gas-liquid) flow data for experiments in which, in the gas phase, the state variables are

$$
(\rho, p, \alpha)=\left(1250 \mathrm{~kg} / \mathrm{m}^{3}, 10^{9} \mathrm{~Pa}, 1\right)
$$

and

$$
(\rho, p, \alpha)=\left(1.2 \mathrm{~kg} / \mathrm{m}^{3}, 10^{5} \mathrm{~Pa}, 1\right)
$$

if $r<r_{1}$ and $r>r_{2}$, respectively, while in the liquid phase they are

$$
(\rho, p, \alpha)=\left(10^{3} \mathrm{~kg} / \mathrm{m}^{3}, 10^{5} \mathrm{~Pa}, 0\right)
$$

if $r_{1}<r \leq r_{2}$, where $r=\sqrt{x^{2}+y^{2}}, r_{1}=0.2 \mathrm{~m}$, and $r_{2}=$ $0.7 \mathrm{~m}$. Here the material quantities of the fluid is taken the same as before, with the exception that a smaller value of the constant for the excluded volume, $b=10^{-4} \mathrm{~m}^{3} / \mathrm{kg}$, is used for the gas.

We note that for this problem all the fluid component is in a resting state initially with zero total velocity, but due to the pressure difference between the fluids at $r=r_{1}$, breaking of the inner circular membrane occurs instantaneously, yielding an outward-going shock wave in liquid, an inward-going rarefaction wave in gas, and a material interface lying in between that separates the gas and liquid. As times go along, the inward-going wave would be reflected from the geometric center that generates a new outward-going wave and induces the subsequent interaction of waves. At a somewhat later time, the outwardgoing shock wave would be collided with the outer gasliquid interface at $r=r_{2}$ that results in a wave pattern consisting of a transmitted shock wave, an interface, and a reflected rarefaction wave. Because of the symmetry of the solution, for simplicity, we only take a quarter of the unit square, and make use of the line of symmetry boundary conditions to the bottom and the left sides during the computations.

Figures 2 and 3 show numerical results for the total density, radial velocity (defined as $\bar{u}=\sqrt{u^{2}+v^{2}}$ ), and pressure, at three stopping times, $t=120,240$, and $450 \mu \mathrm{s}$, where the test has been carried out by using a $200 \times 200$ grid with the high-resolution method. Clearly, from the contour plots shown in Fig. 2, we observe good resolution of the solution structure (i.e., both the shock and interface remain circular and appear to be very well located) after the breaking of the membrane and also the interaction of the shock and the outer interface.

The scatter plots shown in Fig. 3 provide the validation of our two-dimensional results as in comparison with the "true" solution obtained from solving the one-dimensional model with appropriate source terms for the radial symmetry, using the high-resolution method with 1000 mesh points in a unit length domain. That is, for the equation, we have a modified-version of the model (27) in one dimension as

$$
\frac{\partial q}{\partial t}+f\left(\frac{\partial}{\partial r}, q\right)=\psi(q)
$$

with $f$ a vector-value function defined by

$$
\begin{gathered}
f=\left[\frac{\partial}{\partial r}(\rho u), \frac{\partial}{\partial r}\left(\rho u^{2}+p\right), \frac{\partial}{\partial r}(\rho E u+p u),\right. \\
\left.\frac{\partial}{\partial r}\left(\rho_{1} \alpha u\right), u \frac{\partial \alpha}{\partial r}\right]^{T},
\end{gathered}
$$

and $\psi$ the source term derived directly from the geometric simplification of a multidimensional flow to a onedimensional one,

$$
\psi=-\frac{\iota}{r}\left(\rho u, \rho u^{2}, \rho E u+p u, \rho_{1} \alpha u, 0\right)^{T} .
$$

Note that in the case of a $2 \mathrm{D}$ radially or $3 \mathrm{D}$ spherically symmetric flow, we use the quantity $\iota=1$ or 2 , respectively; $u$ now denotes the particle velocity in the $r$-(radial) direction. We use a Strang-type time splitting procedure [38] to deal with the geometric sources of (36) in a high-resolution manner during the run. From the figure, it is clear that our results agree quite well with the "true" solutions at all the selected times, and also free of wrong fluctuations in the pressure near the inner and outer interfaces before and after the interactions of rarefaction and shock waves.

EXAMPLE 4.3. To show how our algorithm performs on shock waves in a more general two-dimensional geometry, we are interested in the simulation of a shock-induced collapse of a cylindrical air cavity in water studied by Bourne 

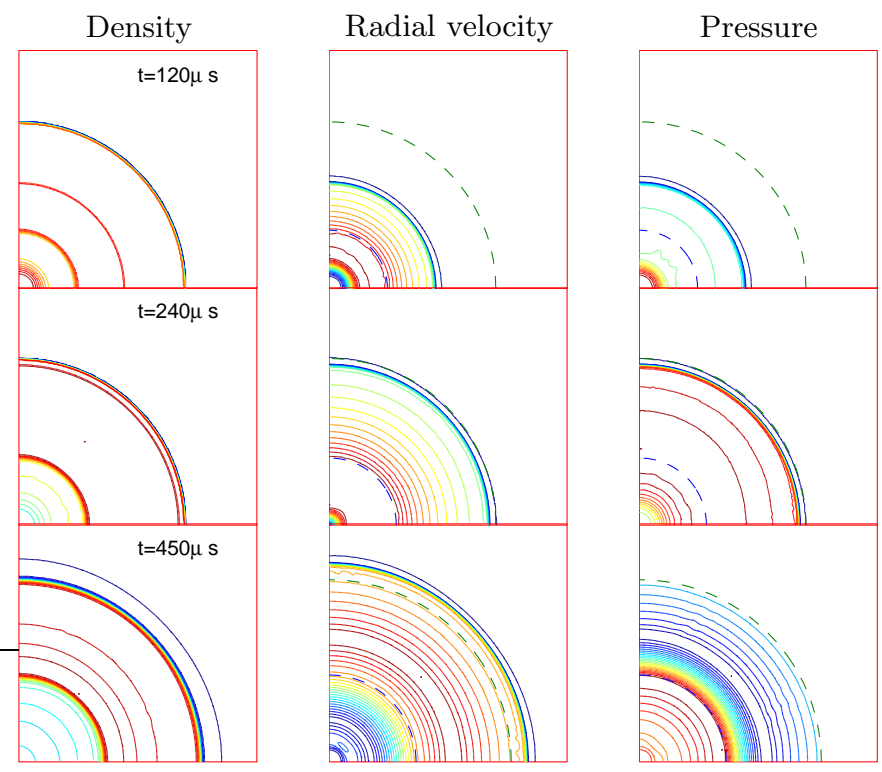

Fig. 2. High-resolution results for a two-phase radially symmetric problem. Contour plots for the total density, radial velocity, and pressure are shown at three different times $t=120$, 240 , and $450 \mu$ s, using a $200 \times 200$ grid. The dashed line shown in the graph is the approximate location of the gas-liquid interface.
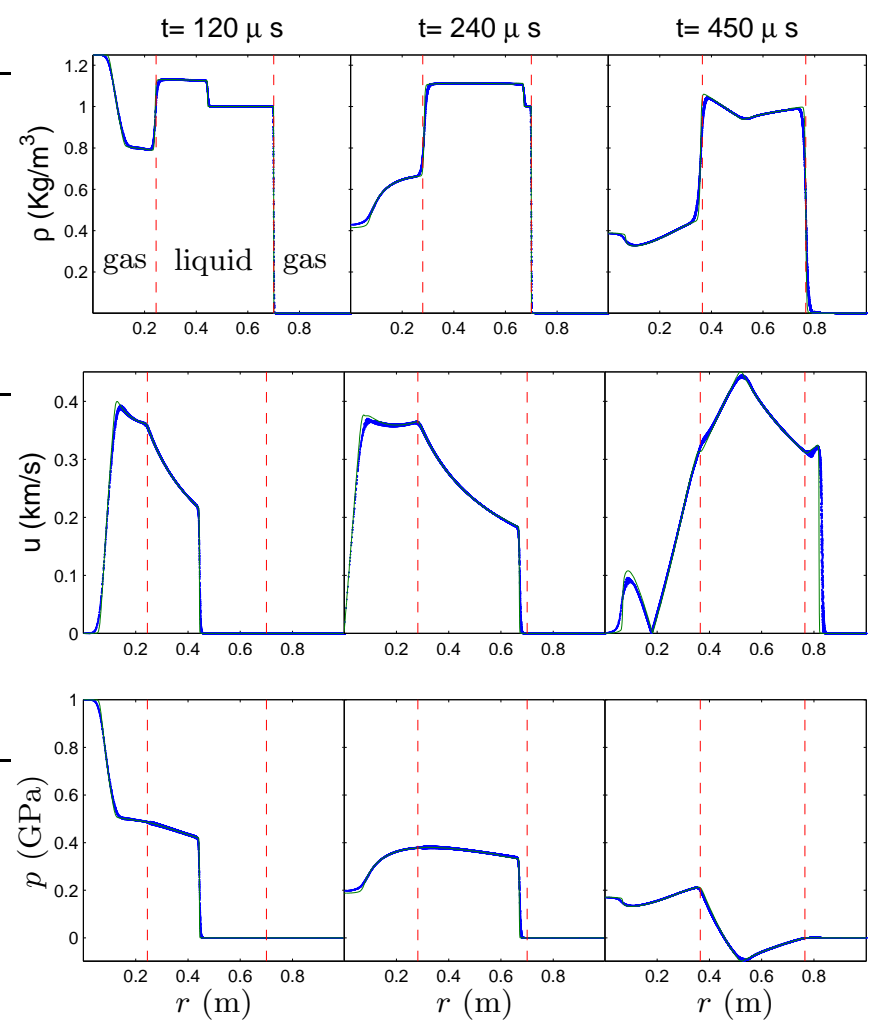

Fig. 3. Scatter plots of the results for the run shown in Fig. 2. The solid line is the "true" solution obtained from solving the one-dimensional model with appropriate source terms for the radial symmetry using the high-resolution method and 1000 mesh points. The dotted points are the two-dimensional result. The dashed line is the approximate location of the gas-liquid interface.

and Field [7] experimentally, and Ball et al. [5] numerically. We setup the problem by introducing a stationary air cavity of radius $r_{0}=3 \mathrm{~mm}$ located at $\left(x_{0}, y_{0}\right)=$ $(6,0) \mathrm{mm}$ and also a planarly rightward-going shock wave in water at $x=1 \mathrm{~mm}$ approaching toward the cavity on the left. Similar to the initial condition employed in ExAMPLE 4.1, inside the air bubble, we use the data on the state $R$ as before, while outside the air bubble (the fluid is water), we use the data on the states $M$ and $L$, when they are in the pre-shock and post-shock regions, respectively. Here, in carrying out the computations below, we take a shock tube of size $[0,15] \times[0,6](\mathrm{mm})^{2}$, and use the solid wall boundary condition on the bottom side, and the non-reflecting boundary condition on the remaining sides.

Figures 4 shows the Schlieren image of the results for the density and pressure at ten different times $t=i \times$ $0.4 \mu \mathrm{s}$, for $i=1,2, \cdots, 10$ (measured relative to a time when the incident shock wave first hits the upstream wall of the air cavity), using the same high-resolution method as in the previous example and a $1500 \times 600$ grid. From the figure, it is easy to see that after the passage of the shock to the cavity, the upstream wall begins to spall across the cavity, yielding an refracted air shock traveling within it at the speed $V_{r} \approx 2.231 \mathrm{~km} / \mathrm{s}$ until its first reflection on the far cavity wall at the time $t \approx 2.647 \mu$ s (see the comments given below on how to obtain these numerical values). Noticing that this upstream cavity wall would involute eventually to form a jet which subsequently crosses the cavity and sends an intense blast wave out into the surrounding liquid upon impacting with the far cavity wall.

Following from [7], the time from the initial impingement of the shock on the upstream wall to the impact of the jet on the downstream wall is referred to as the collapsed time $t_{c}$. In the current case, we find $t_{c} \approx 2.787 \mu \mathrm{s}$, while the experimental result shown in [7] is about $t_{c}=$ $1.8 \pm 0.2 \mu \mathrm{s}$ for an air cavity of the same diameter in gelatine. At a later stage of the collapse, highly compressed air would be trapped in a lobe-like region (see the plots at times $t=3.6$ and $4 \mu \mathrm{s}$ ), where high pressure and also high temperature are concentrated on. It should be mentioned that this lobe-like region is the place where light emission was observed in an experimental work of Bourne and Field [7]. As the time goes along, complex multiple reflection of waves would be continued to happen. The cross section of the results for the same run along the bottom boundary is drawn in Fig. 5 giving some quantitative information about the density and pressure at the selected times. As far as the global wave structure of the solution is concerned the results presented here are reasonable one as compared to the one appearing in Ball et al. [5], where a Free-Lagrange method was employed to solve a similar problem but with a $\gamma$-law gas for the air and an incident shock pressure $1.9 \mathrm{GPa}$; the grid resolution employed in there is $5 \times 10^{4}$ which is 18 times coarser than the grid size used here.

To get a further assessment of the key characteristics of the solutions in the problem, in Fig. 6, we report a diagnosis of the space-time locations of the incident shock wave, the upstream cavity wall, the downstream cavity 
wall, the first refracted shock wave, and the jet, where we again use a $1500 \times 600$ grid and the same numerical method as before. With that, it is a common practice (cf. [22,28]) to perform a linear least-squares fit of these trajectories separately, and take the slope of the respective line as one measure of the speed of $V_{s}, V_{u}, V_{d}, V_{r}$, and $V_{j}$, in the order mentioned. Here, at some sample times, we obtain the positions of the incident and refracted shocks by checking the jumps in the pressure to see whether there are greater than a prescribed tolerance, say $\varepsilon_{p}=\left(p_{L}-p_{M}\right) / 10$, at the horizontal sections of $0.01 \mathrm{~mm}$ below the top boundary, and the axis of symmetry, respectively. As to the positions of the other features, we check the volume fractions to see if there are close to 0.5 at the axis of symmetry, with the exception that, for the upstream wall, we use a section at a height $1.2 \mathrm{~mm}$ above the axis when time $t \geq 1.5 \mu \mathrm{s}$.

For this air-cavity collapse problem in water, it is of great interests to see how the collapse time $t_{c}$ as well as the velocity of the jet are varied as we change the magnitude of the incident shock pressure. Here we carry out one such computation with the set of pressure $p_{L}=i \times 0.5 \mathrm{GPa}$, for $i=1,2, \cdots, 8$, and show the result in Table 1 , observing a monotonic decreasing and so increasing of the collapsed time and the jet velocity, respectively, as the incident shock pressure increases; this is in consistent with the findings shown in Table 1 of [7]. Figure 7 is the graph about the jet velocity together with the Hugoniot locus for the water with the same pre-shock condition as before. Notice that the Hugoniot now divides the graph into two parts where in the lower part the jet velocity is less than the shock velocity and so the incident shock crosses the cavity ahead of the jet, while in the upper part the jet arrives at the downstream cavity wall first and may send out a shock in the material downstream of the cavity ahead of the incident shock. In case the incident shock pressure is in the close neighborhood of 3.5 GPa, the speed of the incident shock would be in the proximity to that of the jet. A figure of this kind has also been displayed in the experimental work of Bourne and Field [7], giving some indication of the sensible behavior of the solutions on the computed jet velocity. It should be remarked that Bagabir and Drikakis [4] have done a similar numerical study, but used the Mach number as the basic parameter for a shock wave in air over a cylindrical helium cavity.

Finally, Fig. 8 shows numerical results for a study of the time history of the volume fraction of the air cavity at four different incident shock pressures $p_{L}=1,2,3$, and 4GPa. As a close look at the data for each of the displayed volume fraction, we may find a time interval during which $\alpha$ falls from 0.8 to 0.1 at almost a linear rate. Consider the case when $P_{L}=2 \mathrm{GPa}$, for example, the time interval for that to occur is $t \in[0.862,2.933] \mu \mathrm{s}$. Our results also show that not long after the collapsed time the volume continuing to decline, but at a somewhat reduced rate which is compatible with many results shown for shockdriven collapse of cylindrical cavities in fluid dynamical problems (cf. $[5,7,12])$. A detailed study of this problem for a spherical air cavity case in three dimensions will be reported elsewhere.
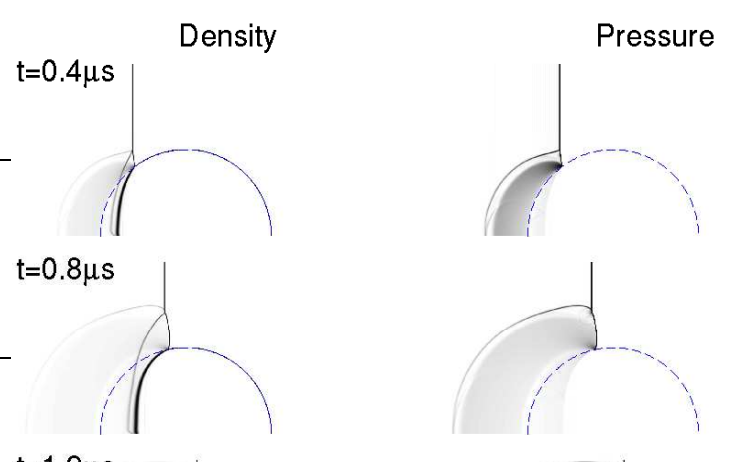

$t=1.2 \mu s$
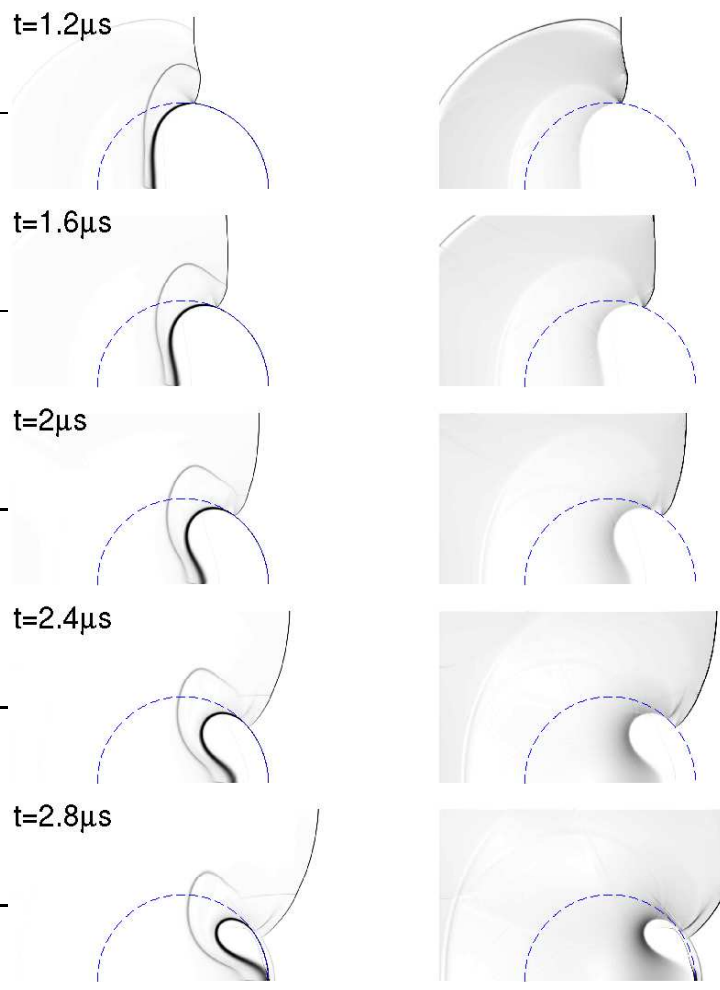

$t=3.2 \mu s$
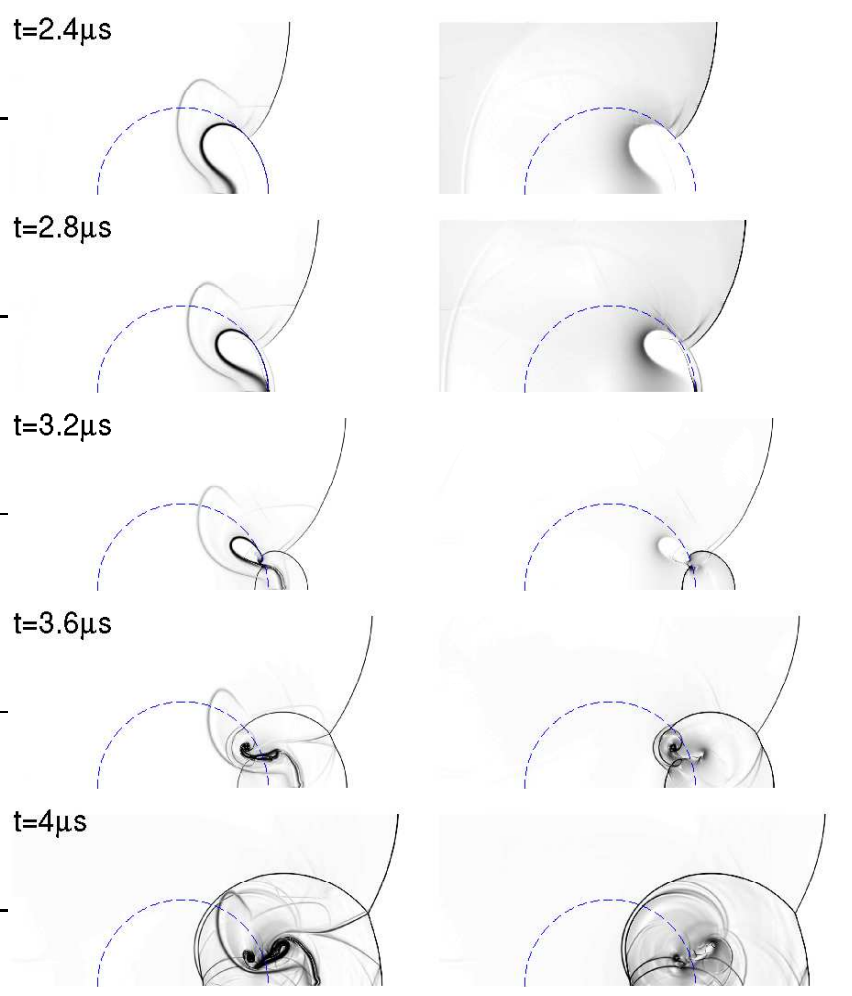

Fig. 4. Two-dimensional results for a planar shock wave in water over a cylindrical air cavity. Schlieren-type image for the density and pressure are shown at ten different times $t=$ $i \times 0.4 \mu \mathrm{s}$, for $i=1,2, \cdots, 10$, where a $1500 \times 600$ grid was used in the computation. The dashed line is the initial location of the air-water interface. 

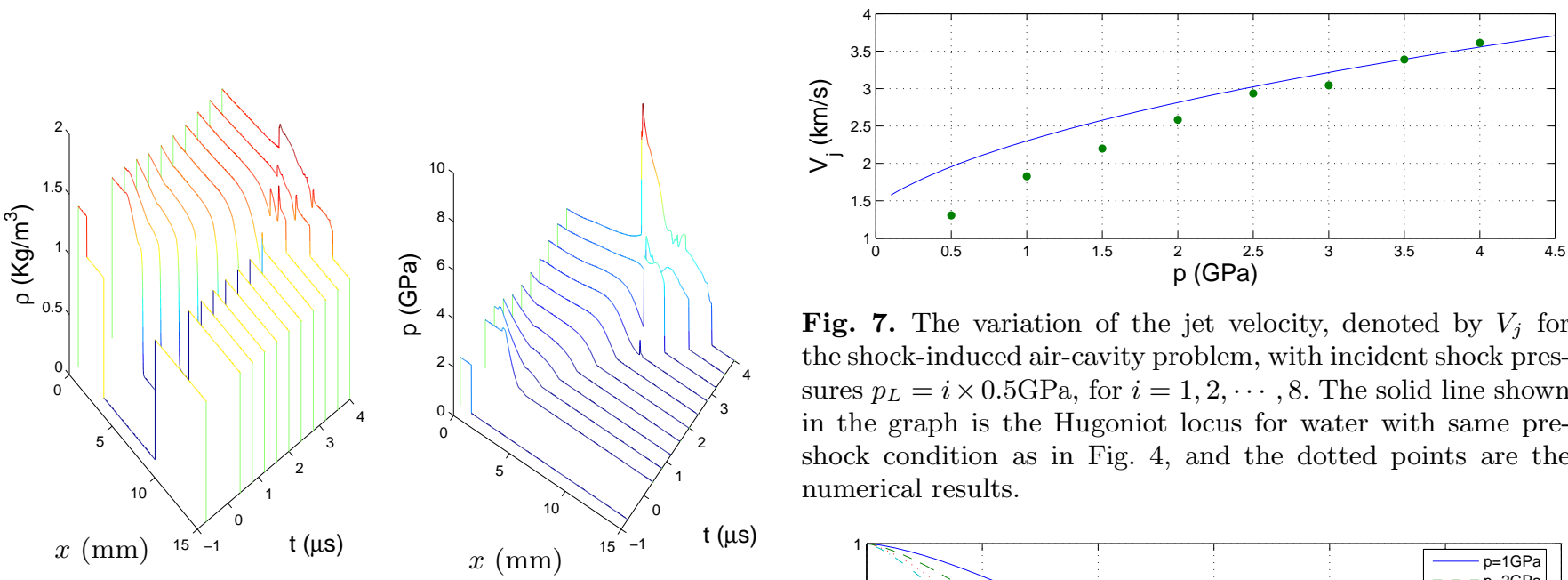

Fig. 7. The variation of the jet velocity, denoted by $V_{j}$ for the shock-induced air-cavity problem, with incident shock pressures $p_{L}=i \times 0.5 \mathrm{GPa}$, for $i=1,2, \cdots, 8$. The solid line shown in the graph is the Hugoniot locus for water with same preshock condition as in Fig. 4, and the dotted points are the numerical results.

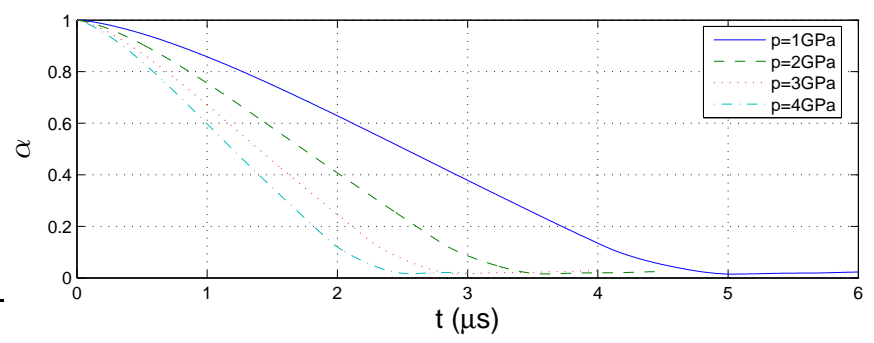

Fig. 8. The time history of the volume fraction of the air cavity at four different incident shock pressures $p_{L}=1,2,3$, and $4 \mathrm{GPa}$.

\section{Conclusions}

Fig. 6. Space-time locations of the incident shock wave, the upstream cavity wall, the downstream cavity wall, the first refracted shock wave, and the jet for the run shown in Fig. 4. These trajectories can be used to estimate the speeds such as $V_{s}, V_{u}, V_{d}, V_{r}$, and $V_{j}$ in the order mentioned above.

Table 1. Data summarizing solutions of a shock-induced air cavity problem for the particle velocity, jet velocity, and collapsed time, at incident shock pressures $p_{L}=i \times 0.5 \mathrm{GPa}$, for $i=1,2, \cdots, 8$

Shock pressure Particle velocity Jet velocity Collapsed time

\begin{tabular}{cccc}
$(\mathrm{GPa})$ & $(\mathrm{km} / \mathrm{s})$ & $(\mathrm{km} / \mathrm{s})$ & $(\mu \mathrm{s})$ \\
\hline 0.5 & 0.256 & 1.309 & 6.091 \\
1.0 & 0.435 & 1.817 & 4.119 \\
1.5 & 0.582 & 2.191 & 3.290 \\
2.0 & 0.711 & 2.572 & 2.787 \\
2.5 & 0.826 & 2.729 & 2.487 \\
3.0 & 0.933 & 3.064 & 2.258 \\
3.5 & 1.032 & 3.350 & 2.072 \\
4.0 & 1.125 & 3.625 & 1.952 \\
\hline
\end{tabular}

We have described an Eulerian interface-capturing approach for a hybrid barotropic and non-barotropic twofluid flow problem in more than one space dimension. The algorithm uses a volume-fraction formulation of the model equations together with an extended equation of state that is devised to ensure a consistent approximation of the energy equation near the numerical-induced smeared material interfaces, and an easy computation of the pressure from the equation of state. A high-order Godunov method based on the wave-propagation viewpoint is employed to solve the proposed model system with the dimensional-splitting technique included in the method for multidimensional problems. Numerical results presented in this paper demonstrate clearly the feasibility of the approach for a reasonable class of two-fluid problems with the thermodynamic property of the barotropic and nonbarotropic fluid components characterized by the Tait and Noble-Abel equations of state, respectively.

Acknowledgement. This work was supported in part by the National Science Council of Republic of China Grants NSC-892115-M-002-033, 90-2115-M-002-022, and 91-2115-M-002-016.

\section{References}

1. Abgrall, R.: How to prevent pressure oscillations in multicomponent flow calculations: a quasi conservative approach. J. Comput. Phys. 125, 150-160 (1996) 
2. Abgrall, R., Nkonga, B., Saurel, R.: Efficient numerical approximation of compressible multi-material flow for unstructured meshes. Computers \& Fluids 32, 571-605 (2003)

3. Allaire, G., Clerc, S., Kokh, S.: A five-equation model for the simulation of interface between compressible fluids. J. Comput. Phys. 181, 577-616 (2002)

4. Bagabir, A., Drikakis, D.: Mach number effects on shockbubble interaction. Shock Waves 11, 209-218 (2001)

5. Ball, G.J., Howell, B.P., Leighton, T.G., Schofield, M.J.: Shock-induced collapse of a cylindrical air cavity in water: a Free-Lagrange simulation. Shock Waves 10, 265-276 (2000)

6. Batten, P., Clarke, N., Lambert, C., Causon, D.: On the choice of wave speeds for the HLLC Riemann solvers. SIAM J. Sci. Comput. 18, 1553-1570 (1997)

7. Bourne, N.K., Field, J.E.: Shock-induced collapse of single cavities in liquid. J. Fluid Mech. 244, 225-240 (1992)

8. Dear, J.P., Field, J.E.: A study of the collapse of arrays of cavities. J. Fluid Mech. 190, 409-425 (1988)

9. Fedkiw, R.P., Aslam, T., Merriman, B.,Osher, S. A nonoscillatory Eulerian approach to interfaces in multimaterial flows (the ghost fluid method). J. Comput. Phys. 152, 457492 (1999)

10. Fermi, E.: Thermodynamics. Dover, New York (1956)

11. Godlewski, E., Raviart, P.A.: Numerical Approximation of Hyperbolic Systems of Conservation Laws. SpringerVerlag, Applied Mathematical Science 118 (1996)

12. Haas, J.F., Sturtevant, B.: Interaction of weak shock waves with cylindrical and spherical gas inhomogeneities. J. Fluid Mech. 181, 41-76 (1987)

13. Harten, A., Lax, P.D., van Leer, B.: On upstream differencing and Godunov-type schemes for hyperbolic conservation laws. SIAM Review 25, 35-61 (1983)

14. Karni, S.: Multicomponent flow calculations by a consistent primitive algorithm. J. Comput. Phys. 112, 31-43 (1994)

15. Koren, B., Lewis, M.R., van Brummelen, E.H., van Leer, B.: Riemann-problem and level-set approaches for homentropic two-fluid flow computations. J. Comput. Phys. 185, 654-674 (2002)

16. Langseth, J.O., LeVeque, R.J.: A wave propagation method for three-dimensional hyperbolic conservation laws. J. Comput. Phys. 165, 126-166 (2000)

17. LeVeque, R.J.: High resolution finite volume methods on arbitrary grids via wave propagation. J. Comput. Phys. 78, 36-63 (1988)

18. LeVeque, R.J.: Wave propagation algorithms for multidimensional hyperbolic systems. J. Comput. Phys. 131, 327-353 (1997)

19. LeVeque, R.J.: Finite Volume Methods for Hyperbolic Problems. Cambridge University Press (2002)

20. Lide, D.R.: Handbook of Chemistry and Physics. CRC Press, 76 edition (1996)

21. Luo, H., Baum, J.D., Löhner, R.: On the computation of multi-material flows using ALE formulation. J. Comput. Phys. 194, 304-328 (2004)

22. Marquina, A., Mulet, P.: A flux-split algorithm applied to conservative models for multicomponent compressible flows. J. Comput. Phys. 185, 120-138 (2003)

23. Marsh, S.P.: LASL Shock Hugoniot Data. University of California Press, Berkeley (1980)
24. Massoni, J., Saurel, R., Nkonga, B., Abgrall, R.: Proposition de méthodes et modèles eulériens pour les problèms à interfaces entre fluides compressibles en présence de transfert de chaleur. Intl. J. Heat and Mass Transfer 45, 12871307 (in french) (2002)

25. Murnaghan, F.D.: Finite Deformation of an Elastic Solid. John Wiley \& Sons, Inc., New York (1951)

26. Murrone, A., Guillard, H.: A five reduced equation model for compressible two-phase flow problems. J. Comput. Phys. 202, 664-698 (2004)

27. Nagoya, H., Obara, T., Takayama, K.: Underwater shock wave propagation and focusing in inhomogeneous media. In Brun, R. et al. (eds.) Proceedings of $19^{\text {th }}$ Intl. Symp. on Shock Waves, Marseille. Springer-Verlag, Berlin, pp. 439-444 (1995)

28. Quirk, J.J., Karni, S.: On the dynamics of a shock-bubble interaction. J. Fluid Mech. 318, 129-163 (1996)

29. Roe, P.L.: Fluctuations and signals- A framework for numerical evolution problems. In Morton, K.W. et al. (eds.) Numerical Methods for Fluid Dynamics. Academic Press, pp. 219-257 (1982)

30. Roe, P.L.: Upwind schemes using various formulations of the Euler equations. In Angrand, F. et al. (eds.) Numerical Methods for the Euler Equations of Fluid Dynamics, SIAM, pp. 14-31 (1985)

31. Saurel, R., Abgrall, R.: A simple method for compressible multifluid flows. SIAM J. Sci. Comput. 21, 1115-1145 (1999)

32. Shyue, K.M.: An efficient shock-capturing algorithm for compressible multicomponent problems. J. Comput. Phys. 142, 208-242 (1998)

33. Shyue, K.M.: A fluid-mixture type algorithm for compressible multicomponent flow with van der Waals equation of state. J. Comput. Phys. 156, 43-88 (1999)

34. Shyue, K.M.: A volume-of-fluid type algorithm for compressible two-phase flows. In Fey, M. et al. (eds.) Hyperbolic Problems: Theory, Numerics, Applications. BirkhäuserVerlag, Intl. Series of Numerical Mathematics, Vol. 130, pp. 895-904 (1999)

35. Shyue, K.M.: A fluid-mixture type algorithm for compressible multicomponent flow with Mie-Grüneisen equation of state. J. Comput. Phys. 171, 678-707 (2001)

36. Shyue, K.M.: A fluid-mixture type algorithm for barotropic two-fluid flow problems. J. Comput. Phys. 200, 718-748 (2004)

37. Smith, R.W.: AUSM (ALE): a geometrically conservative arbitrary Lagrangian-Eulerian flux splitting scheme. J. Comput. Phys. 150, 268-286 (1999)

38. Strang, G.: On the construction and comparison of difference schemes. SIAM J. Numer. Anal. 5, 506-517 (1968)

39. Sweby, P.K.: High resolution schemes using flux limiters for hyperbolic conservation laws. SIAM J. Numer Anal. 21, 995-1011 (1984)

40. Toro, E.F.: A fast Riemann solver with constant covolume applied to the random choice method. Intl. J. Numer. Meth. in Fluids 9, 1145-1164 (1989)

41. Toro, E.F.: Riemann Solvers and Numerical Methods for Fluid Dynamics: A Practical Introduction, 2nd Edition. Springer-Verlag (1999)

42. Toro, E.F., Spruce, M., Speares, W.: Restoration of the contact surface in the HLL-Riemann solver. Shock Waves 4, 25-34 (1994) 
43. van Brummelen, E.H., Koren, B.: A pressure-invariant conservative Godunov-type method for barotropic two-fluid flows. J. Comput. Phys. 185, 289-308 (2003)

44. Wardlaw, Jr., A.B., Mair, H.U.: Spherical solutions of an underwater explosion bubble. Shock and Vibration 5, 89$102(1998)$

45. Yamada,K., Nagoya, H., Takayama, K.: Shock wave reflection and refraction over a two-fluid interface. In Brun, R. et al. (eds.) Proceedings of $19^{\text {th }}$ Intl. Symp. on Shock Waves, Marseille. Springer-Verlag, Berlin, pp. 299-304 (1995) 